\title{
Using an Automatic Tool to Identify Potential Readability Issues in a Large Sample of Medicinal Package Inserts*
}

\author{
C. Pires'; A. Cavaco'; F. Martins'; M. Vigário ${ }^{2}$ \\ ${ }^{1}$ Research Institute for Medicines (iMed.ULisboa), Faculty of Pharmacy, Department of Social Pharmacy, \\ University of Lisbon, Lisbon, Portugal; \\ ${ }^{2}$ Faculty of Letters, Department of Linguistics, University of Lisbon, Lisbon, Portugal
}

Dear Editor,

The main aim of this brief communication was to highlight how automatic methodologies are useful in the process of optimization of medicinal package inserts (PIs). In this context, the objectives of the present work were to quantify the average number of words and the most frequent abbreviations, i.e. the abbreviations with twenty or more occurrences in a large sample of medicinal PIs using an electronic tool that enables to apply an automatic procedure to this end.

PIs of medicinal products are very important in transmitting information on the effective and safe use of medicines [1-4]. Currently, there is a wide range of regulations worldwide concerning the adequate development and approval of PIs by marketing authorization holders and medicine agencies [5-7]. Aiming to ensure proper intelligibility and usability of PIs, it is recommended the use of:

- A limited number of words (e.g. approximately 1500 words) $[4,8-10]$.

- A restricted use of abbreviations, with the exception of the abbreviations to ex-

Correspondence to:

C. Pires, MSc, PhD Student

Research Institute for Medicines (iMed.ULisboa)

Faculty of Pharmacy

Department of Social Pharmacy

University of Lisbon

Avenida Professor Gama Pinto

1170-139 Lisbon

Portugal

E-mail: cmbpires@ff.ul.pt

Methods Inf Med 2015; 54: 379-381

http://dx.doi.org/10.3414/ME15-04-0003

received: March 6, 2015

accepted: May 29, 2015

epub ahead of print: June 25, 2015 press units of measure that are allowed, such as ml, but not cc (cubic centimetres) that may be mistaken as ' $u$ ' (units) $[5-7,11]$.

Although, the number of words varies and is dependent on certain factors, such as the number of the topics focused (e.g. number of indications, contra-indications, etc.), too long PIs are not recommended because patients' comprehension may be affected $[4,8]$. For instance, in a large cross-over study to test the original and optimized version of 5 PIs, with German subjects, it was found that the participants' comprehension and ability to locate information were significantly worse for PIs with more than 1500 words [8]. In the optimized PIs of this study the use of difficult words was reduced in $84 \%$, abbreviations were reduced in $76 \%$, and long words and sentences were reduced in $20 \%$ and $76 \%$, respectively. There are reports highlighting that the users of medicines and health professional reject the use of abbreviations in PIs $[12,13]$ and find that reducing their use in health materials favours PI readability and the safe use of medicines [14]. In another study, the use of Latin abbreviations was particularly difficult to understand even for educated people [15].

Additionally, there are reports of medication errors as a consequence of using certain abbreviations, such as $\mu \mathrm{g}$, mcg and $\mathrm{QD}$, i.e. quaque die (one a day), in the first case, because $\mu \mathrm{g} / \mathrm{mcg}$ were read as $\mathrm{mg}$, in the second case, because in general latin expressions were unknown to the users. Latin expressions are not universally understood. Consequently, the term "microgram" in full and the use of words written in users' mother tongue are recom- mended $[3,5,16]$. Exceptionally, some abbreviations are allowed, such as the abbreviations related to units of measure (e.g. $\mathrm{mg}$ ) or route of administration (e.g. IM for Intramuscular or IV for Intravenous) [5-7, 17].

To the best of our knowledge, no study was previously conducted to assess length and use of abbreviations in the PIs of medicines in use in Portugal. The method followed here may be applied to PIs at use in other countries, including the (type of) software applied.

\section{Settings}

Descriptive study using a large sample of Portuguese package inserts and an automatic methodology.

\section{Methods}

First, approximately $20 \%(n=531)$ of the medicine brands mentioned in the Portuguese Prescribing Guide [18] were randomized. Moreover, this guide describe the majority of the Portuguese medicines. Generic medicines were not included, because the PIs of these medicines are very similar or even equal to the PIs of brand medicines [19].

Second, the PIs of these 531 brands were consulted in Infomed (the public database of Portuguese medicines. Every sampled PI $(\mathrm{n}=531)$ was individually examined in this database (available on www.infarmed.pt/ infomed/inicio.php).

\footnotetext{
Supplementary online material published on our website www.methods-online.com
} 
Third, every abbreviation (defined as contractions of words) was manually identified by direct observation in the sampled PIs. In addition, $10 \%$ of the sampled PIs were randomized and reevaluated in relation to this issue. Since no additional abbreviation was identified using this quality control methodology, it was considered that the manual identification of the abbreviations in the sampled PIs was accurate enough.

Finally, a software application [20] was programmed to automatically count the number of words and abbreviations in each one of the sampled PIs. Exceptionally, a general code for $\mathrm{mg} /$ was created, due to the high number of variations of this abbreviation (e.g. mg/1; mg/0.5, etc.).

The abbreviations with 20 or more occurrences were chosen to illustrate the most common situations. These abbreviations were specifically chosen because their occurrence was above the percentile 90. It was considered that these abbreviations are more likely to be read, thus more relevant to be studied.

\section{Results}

The average number of words per package insert was 1810 (SD = 794; Maximum = 5412; Minimum $=513$ ), with only 217 PIs (40.8\% of 531 ) containing 1500 or less words.

Overall, 38 abbreviations with 20 or more occurrences were identified in the sampled PIs. Moreover, of the 38 abbreviations, $15(39.4 \%)$ represented units of measure, $8(21.1 \%)$ were related to therapeutic groups, 3 (7.9\%) referred to chemical components, and the remaining 12 $(31.6 \%)$ were related to other topics. In these 38 abbreviations, two abbreviations were found with the corresponding full meaning in a language other than Portuguese (HDPE or High-density polyethylene in English and Spp. or Specimen in latin). The full meaning and number of occurrences of the abbreviations that appeared 20 or more times, the number of PIs with these abbreviations and the maximum and minimum number of occurrences of these abbreviations per package insert are pre- sented in $>$ Table 1 ( $>$ online supplementary material).

The most widely used abbreviations were $\mathrm{mg} / \mathrm{ml}$ and EEE (Espaço Económico Europeu 'European economic area'), identified respectively in 102 and 63 PIs. The abbreviations with more occurrences were THS (Hormone replacement therapy) and $\mathrm{mg} / \mathrm{ml}$, respectively appearing 59 and 46 times. To the exception of THS, IM, DC (Coronary heart disease), Spp. (Specimens), and U.I. (International units), all the other abbreviations $(n=33)$ appeared in at least one PI only once (minimum $=1$ ) -Table 1 ( $>$ online supplementary material).

\section{Discussion and Conclusion}

Recent experimental research with German subjects has shown that too long PIs impact on PIs readability, namely those containing more than 1500 words [8]. In the Portuguese PIs under study, a large number of PIs was found that exceed that number. Since it may be expected that patients exposed to excessively long PIs will have problems in locating and understanding information, our results indicate that: i) an effort should be made to shorten the PIs in the Portuguese market; ii) further research is needed on the optimal length of the PIs for the Portuguese population due to the existence of cultural differences and interlinguistic variations [21]; iii) special attention must be given to the way of undertaking a systematic reduction of PIs while maintaining the information that is thought should be available to medicines' users.

Although abbreviations are not recommended in general [5-7], some abbreviations were widely used in our sample of PIs, and in many cases they were non-compliant with the regulatory requirements [7, 17]. For instance, the abbreviations Spp. (Specimen) and HDPE (High-density polyethylene) used in Portuguese PIs, both examples of abbreviations from other languages (the first Latin and the second English), are not universally understood by the target population. On the other hand, there were abbreviations that were compliant with the regulatory requirements [7, 17], such as IV (intravenous) or IM (intramuscular), but that cannot be assumed nonetheless to be understood by PI common users. That most of these abbreviations are in fact not understood by Portuguese potential users of medicines was shown in a recent study [22]. In this study, a convenient sample of Portuguese university undergraduates from humanities studies $(n=10)$ answered to a large questionnaire on the comprehension of scientific abbreviations, such as the abbreviations used in PIs. Of the 1910 abbreviations tested, participants assumed not to know the meaning of 1727 (90.4\%). 113 common abbreviations were also included in the questionnaire as controls. In the case of controls participants reported to known the meaning of the abbreviations and were able to provide their correct meaning in almost all cases $\left(\mathrm{Chi}^{2}=\right.$ 496.2, $\mathrm{p}<0.001$ ). The results of this study clearly indicate that using abbreviations in health written materials is inadequate, and confirm the interest of investigating this topic [22]. We believe that future studies are nevertheless necessary in order to better assess users' interpretation of abbreviations, in different countries and with subjects with varying degrees of literacy.

The abbreviations for units of measure were the most used, as expected, considering the fact that these abbreviations are allowed in the PIs [17]. Additionally, units of measure often used "/" instead of "per" (as in "mg/5 ml" and " $\mathrm{mg} / 2 \mathrm{ml}$ "), in a violation of the requirements of the Institute for Safe Medication Practices List [11].

Moreover, patients are also likely to misinterpret the abbreviations for units of measure [7], or not to understand their meaning (e.g. mmol), which also happens in the case of other technical terms that correspond to unfamiliar words in the common lexica [6].

Other abbreviations frequently used were those referring to chemical elements (e.g. HDPE), as well as EEE (Espaço Económico Europeu 'European economic area'). Abbreviations of the first type are often used in the PI section on the nature and content of the container [4]. However they are specifically not recommended in some regulatory requirements [7]. The use of EEE occurs in the formula This leaflet is available in all EU/EEA languages on the 
European Medicines Agency website [2], which is compulsory in some European PIs.

According to regulations, the exceptional use of abbreviations is associated with their repetition throughout the text [5, 6], but surprisingly it was possible to identify several cases of abbreviation only used once in the sampled PIs. On the other hand, there were several abbreviations repeated frequently, which reinforces the importance of highlighting them when they are used for the first time in the text to encourage a quicker location of their full meaning [5].

Overall, it seems worthwhile to perform comparative analyses between regulations of different countries [5-7], particularly for the marketed medicines with the same PI in different European countries. It is advisable to optimize PIs in terms of length and use of abbreviations, and to update and standardize the regulations on PIs length and use of abbreviations as a way of ensuring their adequacy for each population and cultural background. The development and approval of the PIs should be carefully supervised by the marketing authorization holders and medicines authorities. The use of software such as that used in the present research, may help achieving more accurate and efficient results.

\section{Acknowledgments}

Fundação para a Ciência e a Tecnologia. Ministry of Education and Science. Portugal. The firs author is a doctoral student (Fundação para a Ciência e Tecnologia, http://www.fct.pt/index.phtml.en, Grant: SFRH/BD/76531/2011, Lisbon, Portugal). This project was partially funded by PEst-OE/LIN/UI0214/2013, FCT, Portugal.

\section{Disclosure}

Since this study is part of a larger project, future reports on different data will be considered for publication. In case of future publications, the present Letter will always be cited.

\section{References}

1. Pires C, Vigário M, Cavaco A. Package leaflets of the most consumed medicines in Portugal: safety and regulatory compliance issues. A descriptive study. Sao Paulo Med J 2015; 133 (2): 91-100.

2. European Medicine Agency. Quality Review of Documents Human Product Information Annotated Template version 9. 2013 [consulted on 14/12/2014]. Available at: http://www.ema.europa. eu/docs/en_GB/document_library/Template_or_ form/2009/12/WC500029823.pdf

3. Stahl C, Brauer S, Zeitler HP, et al. How important is a package insert for drug therapy in ambulatory care? J Public Health 2006; 14: 174-177.

4. Wolf A, Fuchs J, Schweim HG. QRD Template Texts Intended for Package Inserts Development from the first QRD template up to the new draft of July 2012. Pharm Ind 2012; 74: 1540 -1549.

5. Food and Drug Administration. Guidance for Industry. Labelling for human Prescription Drug and Biological Products - Implementing the PLR content and Format Requirements; 2013 [consulted on 05/01/2015]. Available at: http://www. fda.gov/downloads/Drugs/GuidanceCompliance RegulatoryInformation/Guidances/ucm075082. pdf

6. European Medicine Agency. Guideline on the readability of the labelling and package leaflet of medicinal products for human use. 2009 [consulted on 05/01/2015]. Available at: http://ec. europa.eu/health/files/eudralex/vol-2/c/2009_01_ 12_readability_guideline_final_en.pdf

7. Australian Commission on Safety and Quality in Health Care. National terminology, abbreviations and symbols to be used in the prescribing and administering of medicines in Australia hospitals. 2006 [consulted on 17/12/2014]. Available at http://www.countryhealthsa.sa.gov.au/LinkClick. aspx?fileticket=MrTKXCi7fEY =

8. Fuchs J. The way forward in package insert user tests from a CRO's perspective. Drug Inf J 2010; 44: 119-129.

9. Institute for Safe Medication Practices. List of Error-Prone Abbreviations, Symbols, and Dose Designations. 2013 [consulted on 17/01/2015]. Available at: http://www.ismp.org/tools/errorpro neabbreviations.pdf
10. Hirsh D, Clerehan R, Staples M, Osborne RH, Buchbinder R. Patient assessment of medication information leaflets and validation of the Evaluative Linguistic Framework (ELF). Patient Educ Couns 2009; 77 (2): 248 -254.

11. Pander Maat H, Lentz L. Improving the usability of patient information leaflets. Patient Educ Couns 2010; 80 (1): $113-119$.

12. Barrio-Cantalejo I, Simón-Lorda P, Jiménez MM, Ruiz AM. Consensus on the legibility criteria of health education leaflets. An Sist Sanit Navar 2011; 34 (2): $153-165$.

13. Pires CM, Cavaco AM. Exploring the perspectives of potential consumers and healthcare professionals on the readability of a package insert: a case study of an over-the-counter medicine. Eur J Clin Pharmacol 2014; 70 (5): 583 -588.

14. Shultz J, Strosher L, Nathoo SN, Manley J. Avoiding Potential Medication Errors Associated with Non-intuitive Medication Abbreviations. Can J Hosp Pharm 2011; 64 (4): 246 -251.

15. Mbuagbaw L, Ndongmanji E. Patients' understanding of prescription instructions in a semiurban setting in Cameroon. Patient Educ Couns 2012; 88 (1): 147-151.

16. Brunetti L, Santell JP, Hicks RW. The impact of abbreviations on patient safety. Jt Comm J Qual Patient Saf 2007; 33: 576-583.

17. European Medicine Agency. Tables of nonstandard abbreviations. 2012 [consulted on 17/12/ 2014]. Available at: http://www.ema.europa.eu/ docs/en_GB/document_library/Regulatory_and_ procedural_guideline/2009/10/WC500004439.pdf

18. Ministry of Health. Prontuário Terapêutico - 10 [Portuguese Prescribing Guide -10]. Lisboa: Infarmed. 2011 [consulted on 10/01/2015]. Available at: http://www.infarmed.pt/portal/page/portal/ INFARMED/PUBLICACOES/PRONTUARIO/ pt10_web.pdf

19. European Commission. Directive 2001/83/EC: Community Code relating to Medicinal products for human use. 2001 [consulted on 17/12/2014]. Available at: http://eur-lex.europa.eu/LexUriServ/ LexUriServ.do?uri=OJ:L:2001:311:0067:0128:en: PDF

20. Martins F, M Vigario, S Frota. PreText - Text Preprocessing. Lisbon: Phonetic Laboratory, CLUL/ FLUL [consulted on 03/05/2015]. Available at: http://labfon.letras.ulisboa.pt/FreP/tools.html

21. Rêgo HH, Braunstein LA, D’Agostino G, et al. When a text is translated does the complexity of its vocabulary change? Translations and target readerships. PLoS One 2014; 9 (10): el10213.

22. Cavaco A, Pires C, Vigário M. Medicine package inserts abbreviations and acronyms in Portuguese: exploring how difficult they are for educated people (Poster, Proceedings from 2013 ICCH. In: Medical Encounter, A Publication of the American Academy on Communication in Healthcare, 2014; 27 (2), 